STRUCTURAL SCIENCE CRYSTAL ENGINEERING MATERIALS

ISSN 2052-5206

Received 3 May 2017

Accepted 22 September 2017

Edited by M. Dusek, Academy of Sciences of the Czech Republic, Czech Republic

Keywords: $\mathrm{Rb}_{2} \mathrm{Ti}_{2} \mathrm{O}_{5} ; \mathrm{C} 2 / m$; band structure.

CCDC references: $1517159 ; 1517160$; 1517161

Supporting information: this article has supporting information at journals.iucr.org/b

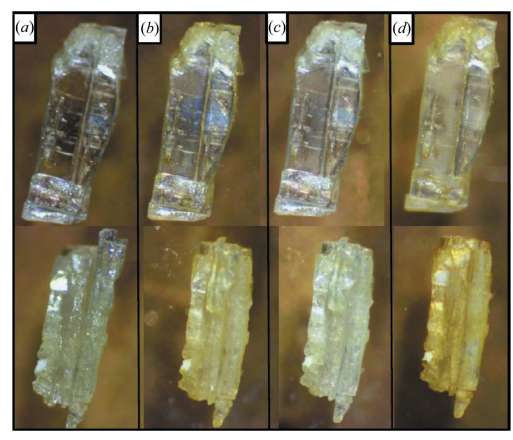

(C) 2017 International Union of Crystallography

\section{The crystal structure of $\mathrm{Rb}_{2} \mathrm{Ti}_{2} \mathrm{O}_{5}$}

\author{
Rémi Federicci, ${ }^{\mathrm{a} *}$ Benoit Baptiste, ${ }^{\mathrm{b}}$ Fabio Finocchi, ${ }^{\mathrm{c}}$ Florin Popa, ${ }^{\mathrm{d}}$ Luc Brohan, ${ }^{\mathrm{d}}$ \\ Keevin Béneut, ${ }^{\text {b }}$ Paola Giura, ${ }^{\text {b }}$ Gwenaëlle Rousse, ${ }^{\text {e,f }}$ Armel Descamps-Mandine, ${ }^{\text {a }}$ \\ Thierry Douillard, ${ }^{\mathrm{g}}$ Abhay Shukla ${ }^{\mathrm{b}}$ and Brigitte Leridon ${ }^{\mathrm{a}}$
}

aLPEM-ESPCI Paris, PSL Research University, CNRS, Sorbonne Universités UPMC, 10 rue Vauquelin, F-75005 Paris France, ${ }^{b}$ IMPMC, Sorbonne Universités UPMC, UMR 7590, CNRS, 4 place Jussieu, F-75005 Paris, France, ' ${ }^{\text {INSP, }}$ Sorbonne Universités UPMC, CNRS, 4 place Jussieu, F-75005 Paris, France, 'Institut des Matériaux Jean Rouxel (IMN), Université de Nantes, CNRS, 2 rue de la Houssinière, BP 32229, F-44322 Nantes Cedex 3, France, ${ }^{\mathbf{e}}$ UMR 8260, "Chimie du Solide et Energie", Collège de France, Sorbonne Universités UPMC, 11 Place Marcelin Berthelot, 75231 Paris Cedex 05, France, 'Réseau sur le Stockage Electrochimique de I'Energie (RS2E), FR CNRS 3459, France, and ${ }^{\mathrm{g} U n i v e r s i t e ́ ~ d e ~ L y o n, ~}$ INSA Lyon, CNRS, MATEIS, UMR 5510, F-69621 Villeurbanne, France. *Correspondence e-mail: remi.federicci@espci.fr

Recent results have demonstrated an exceptionally high permittivity in the range $200-330 \mathrm{~K}$ in crystalline titanium oxide $\mathrm{Rb}_{2} \mathrm{Ti}_{2} \mathrm{O}_{5}$. In this article, the possibility of a structural transition giving rise to ferroelectricity is carefully inspected. In particular, X-ray diffraction, high-resolution transmission electron microscopy and Raman spectroscopy are performed. The crystal structure is shown to remain invariant and centrosymmetric at all temperatures between $90 \mathrm{~K}$ and $450 \mathrm{~K}$. The stability of the $C 2 / m$ structure is confirmed by density functional theory calculations. These important findings allow the existence of a conventional ferroelectric phase transition to be ruled out as a possible mechanism for the colossal permittivity and polarization observed in this material.

\section{Introduction}

The ternary titanium oxides denoted by $M_{2} \mathrm{Ti}_{n} \mathrm{O}_{2 n+1}$ with $M=$ $\mathrm{Li}, \mathrm{Na}, \mathrm{K}, \mathrm{Rb}, \mathrm{Cs}, \mathrm{Fr}$, called the Andersson-Wadsley type alkali titanates (Tournoux et al., 1986), with layered structure for $2 \leq n \leq 5$ and tunnel structure for $6 \leq n \leq 8$, are known for their interesting properties (Shripal et al., 2005). In particular, some of them present ferroelectric phase transitions, with remarkably high Curie temperatures (Vikram et al., 2010).

$M_{2} \mathrm{Ti}_{2} \mathrm{O}_{5} \quad$ (MTO) compounds (Andersson-Wadsley compounds with $n=2$ ) exhibit a fivefold coordination for titanium, which originally came as a surprise because $\mathrm{Ti}^{4+}$ mostly tends to adopt an energetically more stable sixfold coordination (Andersson \& Wadsley, 1960). However, $\mathrm{Ti}^{4+}$ fivefold coordination was reported later in titanite and zirconolite minerals (Farges, 1997), in a natural titanosilicate mineral (Foster et al., 1999), as well as in a few synthetic compounds (Lii, 1995; Roberts et al., 1996). The MTO compounds offer a certain interest for chemistry because of their oxidation abilities (Wang \& Chung, 2009; Wang et al., 2011) but their electrical properties have not been investigated until recently (Federicci et al., 2017) .

Little information has been reported to date in the literature concerning the $\mathrm{Rb}_{2} \mathrm{Ti}_{2} \mathrm{O}_{5}$ (RTO) compound. Early structural investigations were made on $\mathrm{K}_{2} \mathrm{Ti}_{2} \mathrm{O}_{5}(\mathrm{KTO})$, where the space group is reported to be $C 2 / m$ and, more generally, on the MTO family (Andersson \& Wadsley, 1960, 1961; Tournoux et al., 1986). To our knowledge, only two reports exist so far on the synthesis and crystal structure of $\mathrm{Rb}_{2} \mathrm{Ti}_{2} \mathrm{O}_{5}$, which was 
stated to be consistent with the $\mathrm{Cm}$ space group (SchmitzDumont \& Schulz, 1952; Schmitz-DuMont \& Reckhard, 1959).

Recent work (Federicci et al., 2017) on RTO has demonstrated superionic conduction properties stemming from the electrolytic nature of the material due to ionic migration. The ionic conductivity in the temperature range between $200 \mathrm{~K}$ and $330 \mathrm{~K}$ reaches $10^{-3} \mathrm{~S} \mathrm{~cm}^{-1}$, whereas the electronic conduction is five orders of magnitude lower. These two properties were demonstrated to yield important polarization phenomena with colossal relative permittivity reaching $10^{9}$ at low frequency (Federicci et al., 2017). However, as these observations are also consistent with ferroelectric-like behavior, the question of a possible associated structural phase transition arises, as an alternative explanation.

In the present work, we report a systematic characterization of RTO crystal structure as a function of temperature. X-ray diffraction and Raman spectroscopy studies are presented. The experimental results are compared to theoretical calculations of the structural and vibrational properties, performed by density functional theory. The role of vacuum-annealing, which was previously found to be responsible for the 'activation' of the electric properties of the RTO material (Federicci et al., 2017), is also studied and discussed.

\section{Chemical synthesis}

The synthesis of RTO was performed under air by melting $\mathrm{TiO}_{2}$ (rutile) and $\mathrm{RbNO}_{3}$ powders (with purities of $99.95 \%$ ) in a platinium crucible at $1213 \mathrm{~K}$ for $3 \mathrm{~h}$. The heating rate was $5 \mathrm{~K} \mathrm{~min}^{-1}$. The crystals obtained after the slow cooling down process $\left(5 \mathrm{~K} \mathrm{~h}^{-1}\right.$ for $20 \mathrm{~h}$, then $\left.20 \mathrm{~K} \mathrm{~h}^{-1}\right)$ were kept under dry argon atmosphere in order to avoid hydration. The as-grown crystals exhibit a platelet-shape of typically $1 \mathrm{~mm}$ length and hundreds of $\mu \mathrm{m}$ width and thickness.

\section{Characterization}

\subsection{Scanning electron microscopy}

Immediately after the end of the chemical synthesis process, RTO crystals were isolated and scanning electron microscopy (SEM) images were acquired on SEM-FEG at low voltages (from 1 to $5 \mathrm{kV}$ ). Two SEM images which show the platelet shape of two different crystals are displayed in Fig. 1. The growth direction (larger dimension of the crystal) is found to correspond to the $b$ axis. The crystals clearly show a lamellar structure and are shown to be easily cleavable along the $a b$ planes. This aspect may be of interest either for obtaining a very smooth $a b$ surface or a very thin sample.

\subsection{Single-crystal X-ray diffraction}

Single-crystal X-ray diffraction data on RTO were collected on a Bruker Kappa-APEXII four-circle diffractometer equipped with a CCD detector, with Mo $K \alpha$ radiation $(\lambda=$ $0.71073 \AA$, graphite monochromator) at the IPCM X-ray diffraction platform (Université Pierre et Marie Curie, Paris). The measurements were conducted under a $\mathrm{N}_{2}$ flux (Oxford
Cryostream 700) in order to protect the sample from humidity. They were carried out at various temperatures $(400 \mathrm{~K}, 293 \mathrm{~K}$ and $150 \mathrm{~K}$ ) in order to evaluate the stability of the structure. Data reduction, cell refinement, space group determination, scaling, and multiscan absorption correction were performed using CrysAlis PRO software (Rigaku Oxford Diffraction, 2015).

For all data, the structures were solved in the $C 2 / m$ space group by direct methods using SHELXT (Sheldrick, 2015) implemented in Olex2 program (Dolomanov et al., 2009). The refinements were then carried out with SHELXL (Sheldrick, 2015) by full-matrix least-squares minimization and difference Fourier methods. All atoms were refined with anisotropic displacement parameters.

The crystal structure remains unchanged from 150 to $400 \mathrm{~K}$ and the $C 2 / m$ space group was further confirmed by the fact that refinements conducted within noncentrosymmetric space groups did not improve the model nor the reliability factors

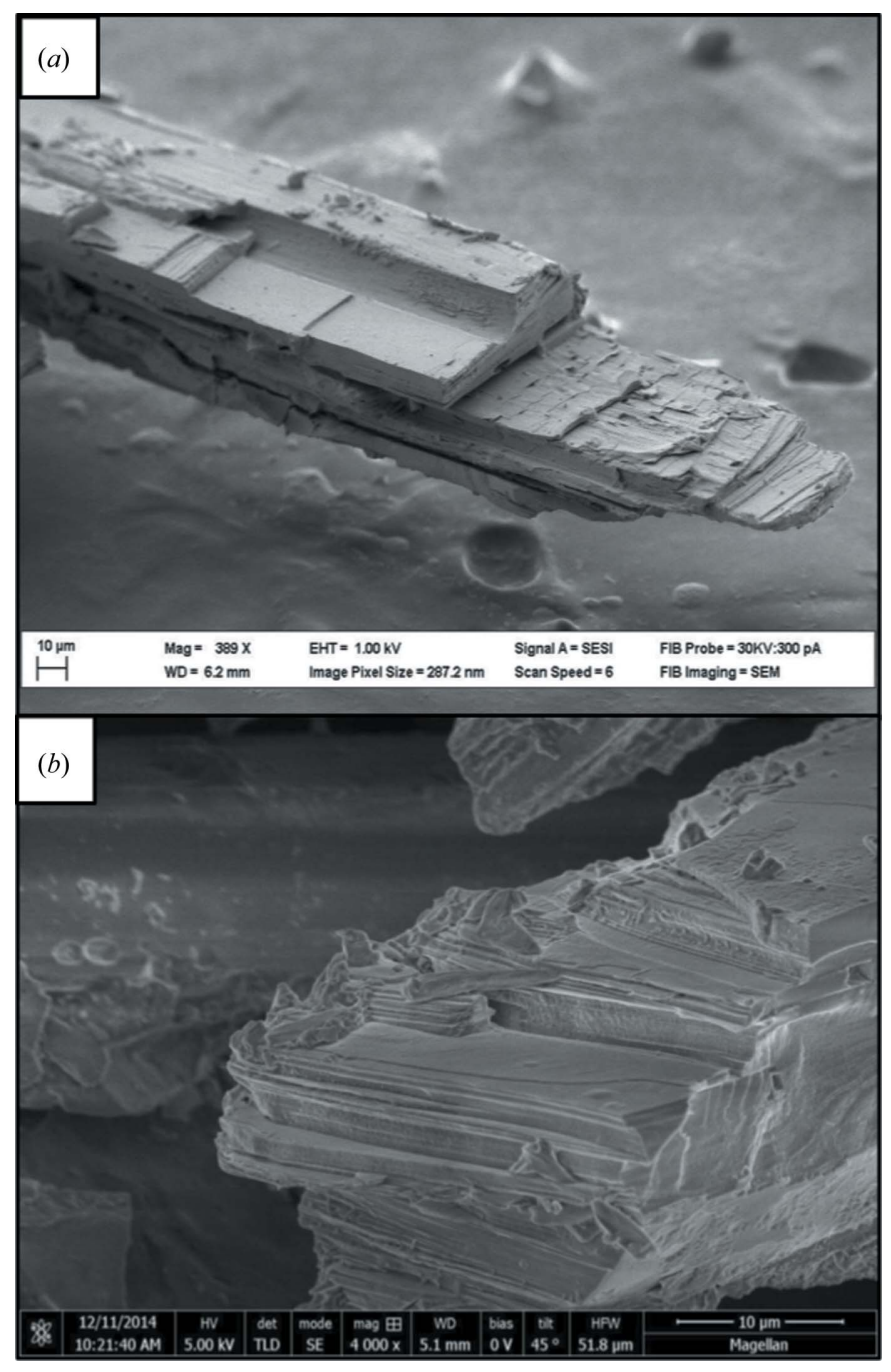

Figure 1

Scanning electron micrographs of two as-grown $\mathrm{Rb}_{2} \mathrm{Ti}_{2} \mathrm{O}_{5}$ crystals from two different batches: $(a)$ magnification $\times 369$ and $(b)$ magnification $\times 4000$. The lamellar structure along the $a b$ planes is clearly evidenced. 
Table 1

Single-crystal X-ray diffraction data for $\mathrm{Rb}_{2} \mathrm{Ti}_{2} \mathrm{O}_{5}$.

For all determinations: $0.5\left(\mathrm{O}_{10} \mathrm{Rb}_{4} \mathrm{Ti}_{4}\right), M_{\mathrm{r}}=346.74$, monoclinic with space group $C 2 / m, Z=2, F(000)=316$. Empirical absorption correction using spherical harmonics, implemented in SCALE3 ABSPACK scaling algorithm.

\begin{tabular}{|c|c|c|c|}
\hline & $400 \mathrm{~K}$ & $293 \mathrm{~K}$ & $150 \mathrm{~K}$ \\
\hline \multicolumn{4}{|l|}{ Crystal data } \\
\hline$a, b, c(\AA)$ & 11.3419 (12), $3.8198(5), 7.0103(8)$ & $11.3370(13), 3.8244(5), 6.9946(8)$ & $11.3457(12), 3.8195(5), 6.9699(8)$ \\
\hline$V\left(\AA^{3}\right)$ & $298.82(6)$ & $298.37(6)$ & $297.12(6)$ \\
\hline$D_{x}\left(\mathrm{Mg} \mathrm{m}^{-3}\right)$ & 3.854 & 3.860 & 3.876 \\
\hline$\mu\left(\mathrm{mm}^{-1}\right)$ & 18.80 & 18.82 & 18.90 \\
\hline \multicolumn{4}{|l|}{ Data collection } \\
\hline Diffractometer & Bruker Kappa-APEXII & Bruker Kappa-APEXII & Bruker Kappa-APEXII \\
\hline Radiation source & Fine focus sealed tube (Mo) & Fine focus sealed tube (Mo) & Fine focus sealed tube (Mo) \\
\hline$T_{\min }, T_{\max }$ & $0.277,1.000$ & $0.323,1.000$ & $0.400,1.000$ \\
\hline $\begin{array}{l}\text { No. of measured, independent and } \\
\text { observed }[I>2 \sigma(I)] \text { reflections }\end{array}$ & $1859,567,511$ & $1389,351,333$ & $1205,348,320$ \\
\hline$R_{\text {int }}$ & 0.023 & 0.021 & 0.041 \\
\hline$R\left[F^{2}>2 \sigma\left(F^{2}\right)\right], w R\left(F^{2}\right), S$ & $0.035,0.092,1.08$ & $0.028,0.073,1.17$ & $0.050,0.134,1.11$ \\
\hline No. of reflections & 567 & 351 & 348 \\
\hline No. of parameters & 30 & 29 & 29 \\
\hline$\Delta \rho_{\max }, \Delta \rho_{\min }\left(\mathrm{e} \AA^{-3}\right)$ & $0.82,-1.63$ & $0.68,-1.45$ & $1.52,-1.93$ \\
\hline
\end{tabular}

Table 2

Fractional atomic coordinates $\left(\times 10^{4}\right)$ and equivalent isotropic displacement parameters $\left(\AA^{2} \times 10^{3}\right)$.

$U_{\text {eq }}$ is defined as $1 / 3$ of the trace of the orthogonalised $\mathbf{U}_{I J}$ tensor.

\begin{tabular}{llllll}
\hline & Atom & $x$ & $y$ & $z$ & $U(\mathrm{eq})$ \\
\hline \multirow{2}{*}{$400 \mathrm{~K}$} & Rb1 & $988.8(5)$ & 5000 & $8486.6(7)$ & $26.7(2)$ \\
& Ti1 & $3525.6(6)$ & 5000 & $5883.7(11)$ & $12.6(2)$ \\
& O1 & 5000 & 5000 & 5000 & $24.5(11)$ \\
& O2 & $3763(4)$ & 5000 & $8355(5)$ & $25.7(8)$ \\
& O3 & $1780(3)$ & 5000 & $4786(5)$ & $17.4(7)$ \\
$293 \mathrm{~K}$ & Rb1 & $985.1(5)$ & 5000 & $8481.2(8)$ & $21.0(3)$ \\
& Ti1 & $3524.9(8)$ & 5000 & $5884.9(14)$ & $10.8(3)$ \\
& O1 & 5000 & 5000 & 5000 & $19.7(13)$ \\
& O2 & $3762(4)$ & 5000 & $8367(6)$ & $20.7(9)$ \\
& O3 & $1780(3)$ & 5000 & $4797(5)$ & $14.3(8)$ \\
$150 \mathrm{~K}$ & Rb1 & $981.2(8)$ & 5000 & $8472.5(12)$ & $14.9(5)$ \\
& Ti1 & $3522.9(14)$ & 5000 & $5897(2)$ & $9.7(5)$ \\
& O1 & 5000 & 5000 & 5000 & $15(2)$ \\
& O2 & $3767(6)$ & 5000 & $8391(9)$ & $15.6(15)$ \\
& O3 & $1772(6)$ & 5000 & $4776(9)$ & $14.9(14)$ \\
\hline
\end{tabular}

whatever the temperature. Moreover, the atomic displacement parameters did not reveal any suspect elongated shape that would have been a sign of atomic positions incompatible with the $C 2 / m$ space group. The single-crystal data parameters and the atomic positions at $400 \mathrm{~K}, 293 \mathrm{~K}$ and $150 \mathrm{~K}$ are reported in Tables 1 and 2, respectively. (The reliability factors obtained from refinement of data collected at low temperature are less satisfactory due to the likely fragmentation of the crystal during the cooling.)

The unit cell of RTO is shown in Fig. 2 using VESTA software (Momma \& Izumi, 2011). This two-dimensional structure consists in alternate layers of Ti-O (namely $\mathrm{Ti}_{2} \mathrm{O}_{5}^{2-}$ ) and $\mathrm{Rb}$ (namely $\mathrm{Rb}^{+}$) atoms stacked along the $c$ axis. The Ti$\mathrm{O}$ layers correspond to planes of pyramids pointing up or down and sharing either corners or edges. The Ti atoms are at the center of these slightly tilted square-based-pyramids and are therefore surrounded by five oxygen atoms.

The structure is consistent with the space group $C 2 / m$ and identical for all the measured temperatures. The volume as well as the cell parameter $c$ are found to decrease with decreasing temperature, as expected. The pyramid apical

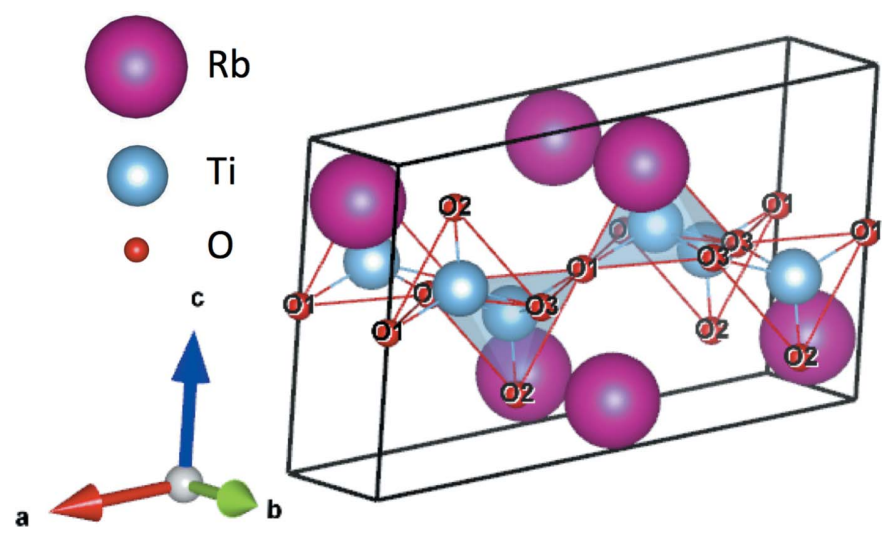

Figure 2

Structure of $\mathrm{Rb}_{2} \mathrm{Ti}_{2} \mathrm{O}_{5}$ as obtained from the XRD measurements and visualized using VESTA software. The corresponding space group is $C 2 / \mathrm{m}$. Please note the lamellar character of the structure, the alternate downward and upward pyramids and the fivefold coordination for $\mathrm{Ti}$ atoms. 
angle $\mathrm{O} 1-\mathrm{O} 2-\mathrm{O} 3$ (within the $b c$ plane) decreases linearly from $400 \mathrm{~K}$ to $150 \mathrm{~K}$ of about $0.3^{\circ}$. The distance between the apical oxygen and the titanium atom increases with decreasing temperature. This tends to indicate that the pyramid is more elongated at lower temperature. Of course this would deserve further confirmation by a more systematic study including more temperature points.

\subsection{Powder X-ray diffraction}

The X-ray powder diffraction (XRD) patterns were recorded at the IMPMC X-ray diffraction platform using an XPert Pro Panalytical diffractometer equipped with a $\mathrm{Cu} K \alpha$ radiation source $\left(\lambda K \alpha_{1}=1.54056 \AA, \lambda K \alpha_{2}=1.54439 \AA\right)$ with an XCelerator detector. The measurements were conducted under nitrogen atmosphere (under vacuum for $100 \mathrm{~K}$ acquisition) in an Anton Paar HTK 450 temperature-controlled chamber. Rietveld refinements (Rietveld, 1969) were performed with the FullProf suite of programs (RodriguezCarvajal, 1993) on X-ray powder diffraction patterns recorded in Bragg-Brentano geometry. The background was modeled using a 12-coefficient-polynome, and Thomson-Cox-Hasting

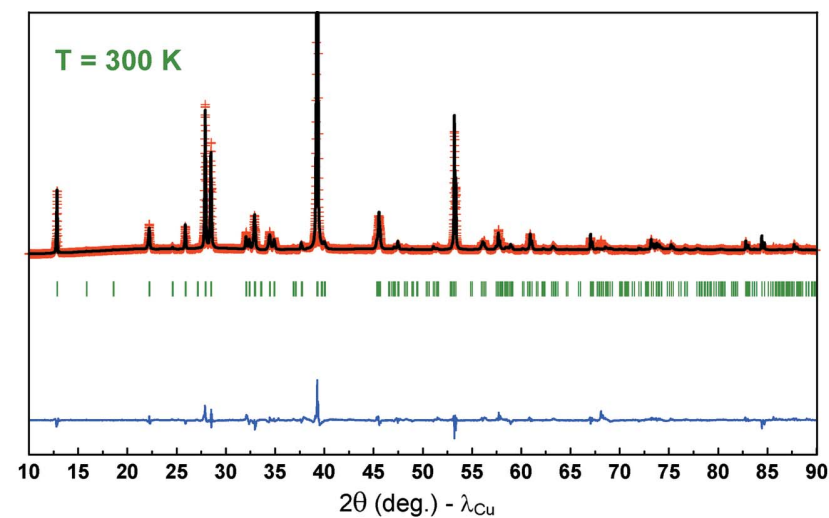

(a)

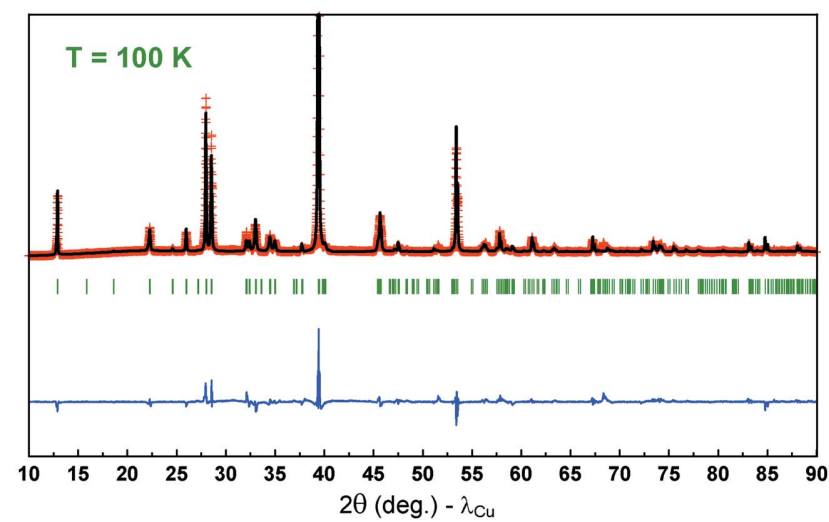

(b)

Figure 3

Rietveld refinements and $\mathrm{Rb}_{2} \mathrm{Ti}_{2} \mathrm{O}_{5}$ powder X-ray diffraction patterns (Cu wavelength) at $300 \mathrm{~K}$ (top) and $100 \mathrm{~K}$ (bottom). The red circles are the experimental points, the black line is the calculated pattern, the green vertical ticks refer to Bragg reflections and the blue line is the difference between observed and calculated patterns. Similar diffractograms at $200 \mathrm{~K}$ and $400 \mathrm{~K}$ are displayed in the supporting information. The diffractograms are identical at all temperatures, ruling out strong evidence for a structural transition.
Table 3

Results of the Rietveld refinement on the X-ray powder patterns of $\mathrm{Rb}_{2} \mathrm{Ti}_{2} \mathrm{O}_{5}$ at different temperatures.

\begin{tabular}{lllll}
\hline & $400 \mathrm{~K}$ & $300 \mathrm{~K}$ & $200 \mathrm{~K}$ & $100 \mathrm{~K}$ \\
\hline Space group & $C 2 / m$ & $C 2 / m$ & $C 2 / m$ & $C 2 / m$ \\
$a(\AA)$ & $11.34378(2)$ & $11.34545(2)$ & $11.34381(2)$ & $11.34265(3)$ \\
$b(\AA)$ & $3.82905(6)$ & $3.82824(5)$ & $3.82756(5)$ & $3.82557(6)$ \\
$c(\AA)$ & $7.00371(5)$ & $6.99109(5)$ & $6.98718(5)$ & $6.96969(6)$ \\
$\beta\left({ }^{\circ}\right)$ & $100.2413(6)$ & $100.2889(5)$ & $100.2939(6)$ & $100.3379(7)$ \\
$V\left(\AA^{3}\right)$ & $299.365(8)$ & $298.762(7)$ & $298.495(7)$ & $297.521(9)$ \\
$R_{\text {Bragg }}\left({ }^{\circ}\right)$ & 13.4 & 9.86 & 12.1 & 13 \\
\hline
\end{tabular}

Voigt functions (Finger et al., 1994) were used as peak shape functions. Preferred orientation was taken into account in the refinements, as all (001) reflections were overestimated. Measurements in a capillary would have limited this effect but they were not possible because of the Bragg Brentano (reflection) geometry imposed by our cryostat. The diffractograms collected on RTO are displayed in Fig. 3 for different temperatures ranging between $100 \mathrm{~K}$ and $400 \mathrm{~K}$. Table 3 gives the lattice parameters obtained from the Rietveld refinements performed on the X-ray powder patterns that are also shown in Fig. 3, with $\mathrm{Cu} K \alpha$ spectroscopic wavelength.

An overall zero-shift parameter and lattice parameters were refined, together with profile parameters and atomic positions.

The atomic positions and lattice parameters extracted from the single-crystal and the powder measurements are found to be consistent and allow the $C 2 / m$ structure to be established, over the investigated range of temperature. The refinements reveal that the single-crystal structure is representative of the whole sample and further confirm the absence of structural transition from $100 \mathrm{~K}$ to $400 \mathrm{~K}$. They also demonstrate that the structure is identical to the one previously reported for $\mathrm{K}_{2} \mathrm{Ti}_{2} \mathrm{O}_{5}$ (Andersson \& Wadsley, 1961).

\subsection{High-resolution transmission electron microscopy}

High-resolution transmission electron micrographs (HRTEM) were recorded at INSA (Lyon) for a RTO sample (using a HR-TEM JEM-2010F from JEOL Ltd, Tokyo, Japan). The TEM lamella were prepared using a $\mathrm{Ga}^{2+}$ focussed ion beam (NVision 40 from Carl Zeiss, Oberkochen, Germany). Fig. 4 allows us to assess the very good crystallinity of the crystal on a local scale.

The inset of Fig. 4 shows an enlargement of a particular region of the image. An overlay of the crystal structure as calculated from XRD data (as described above) is displayed on top of the micrograph, both in excellent agreement. This provides an additional confirmation that the $C 2 / \mathrm{m}$ structure calculated from the XRD measurement is correct.

\section{Raman spectroscopy}

Raman spectroscopy measurements were performed on RTO crystals over the range of temperature $90-450 \mathrm{~K}$ under controlled atmosphere conditions. The set-up consisted of an argon laser source (Spectra-physics Argon 514.5nm), a spec- 


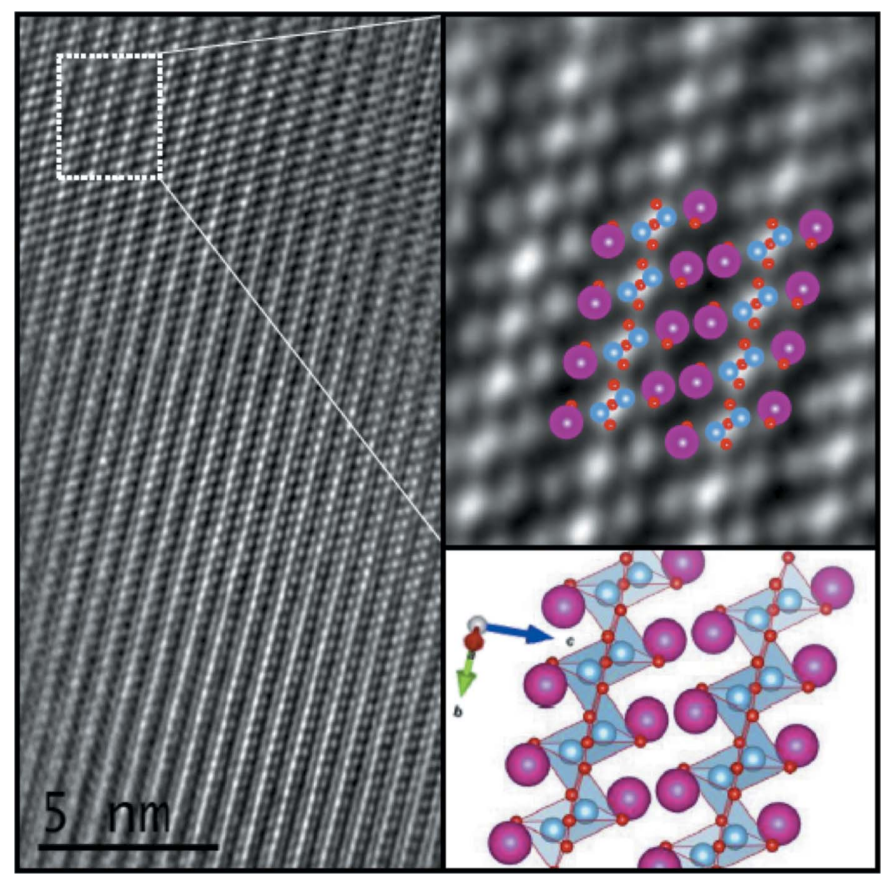

Figure 4

High-resolution transmission electron micrograph of an $\mathrm{Rb}_{2} \mathrm{Ti}_{2} \mathrm{O}_{5}$ sample along the [110] axis. A magnification of the image contained within the white rectangle is displayed in the inset with superposition of a simulated crystal structure obtained from X-ray diffraction data. The color codes for the atoms are the same as for Fig. 2.

trometer (T64000 HORIBA Jobin Yvon), a set of optical microscopy lens in order to focus the laser beam over a few micrometers and a cryostat (Linkham FTIR 600) used to control the helium atmosphere and the temperature. The power of the laser beam was set to $140 \mathrm{~mW}$ and the recording times to $30 \mathrm{~s}$. Each Raman spectrum is an average from three distinct spectra acquired in a row.

Raman spectra recorded between $90 \mathrm{~K}$ and $450 \mathrm{~K}$ over the range $180-1300 \mathrm{~cm}^{-1}$ are plotted in Fig. 5. As-grown samples that had been kept under dry atmosphere were used. The measurements were performed in the following order: $300 \mathrm{~K}$, then cooling down to $240 \mathrm{~K}, 200 \mathrm{~K}, 140 \mathrm{~K}, 90 \mathrm{~K}$ and then warming up to $450 \mathrm{~K}$. The spectra remain qualitatively

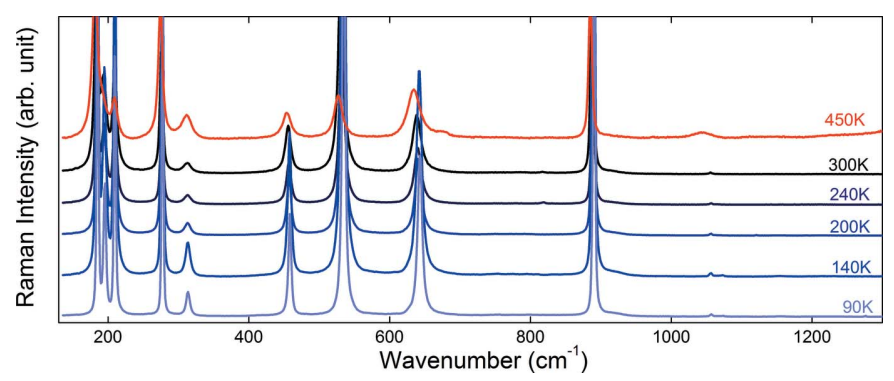

Figure 5

Experimental Raman spectra obtained on an $\mathrm{Rb}_{2} \mathrm{Ti}_{2} \mathrm{O}_{5}$ as-grown crystal as function of the temperature under helium atmosphere between $180 \mathrm{~cm}^{-1}$ and $1300 \mathrm{~cm}^{-1}$. The first spectrum is taken at $300 \mathrm{~K}$, then the following spectra are collected at $240 \mathrm{~K}, 200 \mathrm{~K}, 140 \mathrm{~K}$ and $90 \mathrm{~K}$, then the last spectrum is taken at $450 \mathrm{~K}$. No significative changes occur with temperature variation. unchanged when temperature is varied between $300 \mathrm{~K}$ and $90 \mathrm{~K}$, which attests to the stability of the structure over this range of temperatures. However some small features at around $300 \mathrm{~cm}^{-1}$ and $1000 \mathrm{~cm}^{-1}$ are seen to vary as function of temperature. (This may be related to some distortion in the structure due either to temperature or to small displacement of the laser beam with temperature but does not question the $C 2 / m$ structure.)

This Raman spectroscopy investigation represents therefore a second probe attesting to the overall stability of the centrosymmetric $C 2 / m$ crystal structure of RTO between $90 \mathrm{~K}$ and $300 \mathrm{~K}$.

\section{Density functional theory calculations \\ 5.1. Crystal structure}

The structural calculations were performed on the RTO unit cell, which consists of two formula units, through the Quantum Espresso package (Giannozzi et al., 2009) that is based on the density functional theory (DFT). Both local density approximation (LDA) and generalized gradient approximation (GGA) functionals were used (Perdew et al., 1996). We employed ultra-soft pseudopotentials, which include semi-core states for $\mathrm{Rb}$ and Ti. A Monkhorst-Pack $2 \times 6 \times 3$ grid and a cutoff energy of 50 Ryd for the expansion of the Kohn-Sham (Hohenberg \& Kohn, 1964; Kohn \& Sham, $1965)$ orbitals in plane waves $(4 \times$ as large for the charge density and potential) were enough to obtain the converged energies and the structural parameters.

The initial conditions of the structural optimization are the following: the space group was set to $C 2 / m$ and the atomic positions were those extracted from the X-ray diffraction measurements, which are given in Table 2. The lattice parameters, angles and atomic positions were then left free during the calculation. Their final values, within either the GGA or the LDA approximations, are collected in Table 4. A series of structural optimizations have been conducted at several volumes around the equilibrium volume. The Murnaghan equation of state (Murnaghan, 1944) was used to extract the four parameters: the equilibrium volume $V_{0}$, the equilibrium internal energy $E_{0}$, the bulk modulus $B_{0}$ and its pressure derivative $B_{0}{ }^{\prime}$, that are all given in Table 4 .

The computed atomic positions are reported in Table 5. We see that the structural parameters that were computed within the GGA approximation are extremely close to their experimental counterparts that are collected in Table 1. The computed enthalpy of formation of RTO at zero pressure from the elementary stable phases $\mathrm{Rb}_{(\mathrm{s})}, \mathrm{Ti}_{(\mathrm{s})}$ and $\mathrm{O}_{2 \text { (gas) }}$ is $-309.8 \mathrm{~kJ}^{-1}$, within this approximation. These theoretical calculations thus confirm that the structure is thermodynamically stable and can be predicted with a high level of accuracy.

\subsection{Band structure}

The band structure pictured in Fig. 6 is calculated using the GGA functional for the corresponding optimized crystal 
Table 4

Computed equilibrium lattice constants within the GGA and the LDA.

GGA: generalized gradient approximation; LDA: local density approximation. The equilibrium volume $V_{0}$, bulk modulus $B_{0}$ and its pressure derivative $B_{0}{ }^{\prime}$ have been obtained through a fit to the Murnaghan equation of state (see text).

\begin{tabular}{lcc}
\hline & GGA & LDA \\
\hline$a(\AA)$ & 11.3318 & 11.1425 \\
$b(\AA)$ & 3.8322 & 3.7492 \\
$c(\AA)$ & 6.9863 & 6.7665 \\
$\alpha\left(^{\circ}\right)$ & 90 & 90 \\
$\beta\left(^{\circ}\right)$ & 100.110 & 100.327 \\
$\gamma\left({ }^{\circ}\right)$ & 90 & 90 \\
$V_{0}(\AA)$ & 298.688 & 278.105 \\
$E_{0}(\mathrm{Ryd})$ & $-980.1266(6)$ & $-980.4192(1)$ \\
$B_{0}(\mathrm{GPa})$ & $52.6(4)$ & $71.0(2)$ \\
$B_{0}{ }^{\prime}$ & $8.8(6)$ & $4.6(3)$ \\
\hline
\end{tabular}

structure. The path used along the Brillouin zone is chosen using the MCL structure (Setyawan \& Curtarolo, 2010). A gap of at least $3.54 \mathrm{eV}$, which is likely to be underestimated as usual in the DFT-GGA, attests to the insulating nature of the RTO crystal.

\subsection{Phonon modes}

Phonon mode calculations are based on the density functional perturbation theory (Baroni et al., 2001). These calculations were performed by using LDA or GGA functionals and carried out for the corresponding relaxed structures. The phonon frequencies at the center of the Brillouin zone were computed with ultrasoft pseudopotentials, whereas the Raman intensities were estimated by employing norm-conserving pseudopotentials on top of the phonon calculations, and are therefore less accurate than the frequencies themselves. The theoretical phonon spectra are convolved with Lorentzians and plotted in Fig. 7. The Raman intensities and the phonon

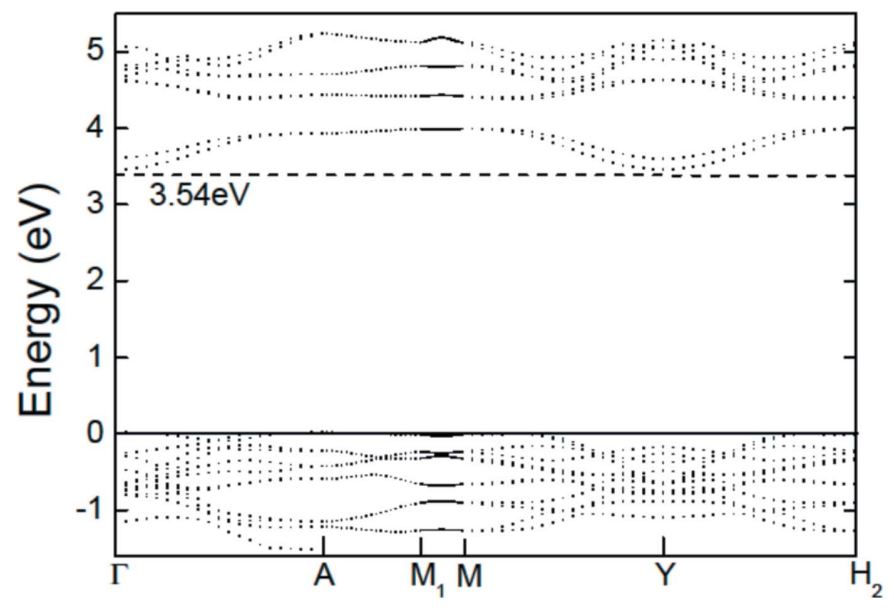

Figure 6

Band structure of $\mathrm{Rb}_{2} \mathrm{Ti}_{2} \mathrm{O}_{5}$ calculated with GGA functional (see text). The high-symmetry points are chosen following the path of the MCL structure (Setyawan \& Curtarolo, 2010). The highest occupied electronic level in the valence band is set at zero, while the dashed line marks the lowest electronic level in the conduction band.
Table 5

Fractional atomic coordinates $\left(\times 10^{4}\right)$ after structural relaxation for the $\mathrm{Rb}_{2} \mathrm{Ti}_{2} \mathrm{O}_{5}$ crystal, within the GGA.

\begin{tabular}{llll}
\hline Atom & $x$ & $y$ & $z$ \\
\hline $\mathrm{Rb}$ & 1003 & 5000 & 8493 \\
$\mathrm{Ti}$ & 3524 & 5000 & 5912 \\
$\mathrm{O} 1$ & 5000 & 5000 & 5000 \\
$\mathrm{O} 2$ & 3754 & 5000 & 8469 \\
$\mathrm{O} 3$ & 1769 & 5000 & 4811 \\
\hline
\end{tabular}

frequencies are extracted from the DFT calculations whereas the full width at half-maximum is arbitrarily set to 1 in order to render them discernible.

With respect to the experimental data, the computed frequencies are higher with the LDA and lower with the GGA. This can be related to the fact that the LDA functional generally underestimates the lattice parameters and provides stiffer force constants, whereas the GGA does the reverse. When looking at the high-frequency part of the spectrum, the four phonon modes are bracketed by the theoretical phonon modes found with GGA and LDA functionals, which differ by not more than $56 \mathrm{~cm}^{-1}$. These four phonon modes are denoted $\nu 1, v 2, v 3$ and $v 4$, from small to high frequencies. The representations of these four phonon modes are sketched in Fig. 8. They all correspond to vibrational modes of the $\mathrm{Ti}-\mathrm{O}$ bonds.

At low frequencies, ten theoretical modes were computed between $80 \mathrm{~cm}^{-1}$ and $330 \mathrm{~cm}^{-1}$. They can be unambiguously related to the set of experimental modes in the same wavenumber range, even though their intensities do not match with the experimental ones. The representations of all the calculated phonon modes are displayed in the supplementary material. The good agreement between the calculated phonon spectrum for the simulated RTO system and the Raman spectrum obtained experimentally on a RTO crystal confirms that each mode can be assigned and analyzed in terms of a consistent set of atomic displacements and excludes the presence of singularities.

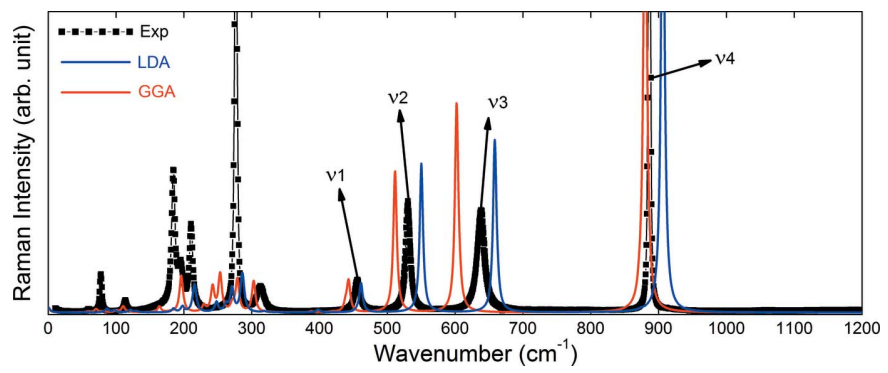

Figure 7

Experimental and simulated Raman spectra for $\mathrm{Rb}_{2} \mathrm{Ti}_{2} \mathrm{O}_{5}$. The black squares represent the experimental Raman spectrum data points, between $10 \mathrm{~cm}^{-1}$ and $1200 \mathrm{~cm}^{-1}$ for a crystal of $\mathrm{Rb}_{2} \mathrm{Ti}_{2} \mathrm{O}_{5}$ measured at $300 \mathrm{~K}$. The blue and red curves are the theoretical Raman spectra simulated with DFT using respectively LDA and GGA functionals. The agreement is very good, as may be seen for the $v 1, v 2, \nu 3$ and $v 4$ phonon modes, taking into account the tendencies of LDA and GGA to respectively overestimate and underestimate the phonon frequencies. This confirms that the structure is consistent with the centrosymmetric $C 2 / m$ space group. The indicated $\nu 1, v 2, \nu 3$ and $\nu 4$ phonon modes are represented in Fig. 8. 
The computed diagonal effective charges for $\mathrm{Ti}$ are $Z_{x x}^{*}=4.71, Z_{y y}^{*}=4.72, Z_{z z}^{*}=3.08$, with much smaller offdiagonal elements $Z_{x z}^{*}$ and $Z_{z x}^{*}$. In contrast to typical ferroelectric crystals such as $\mathrm{BaTiO}_{3}$ (Rabe \& Ghosez, 2007), the effective charges do not show a relevant anomalous contribution, which points toward the absence of an electricallydriven instability in RTO.

\section{Role of vacuum annealing}

As reported earlier (Federicci et al., 2017), the remarkable electrical properties of RTO are strongly enhanced for

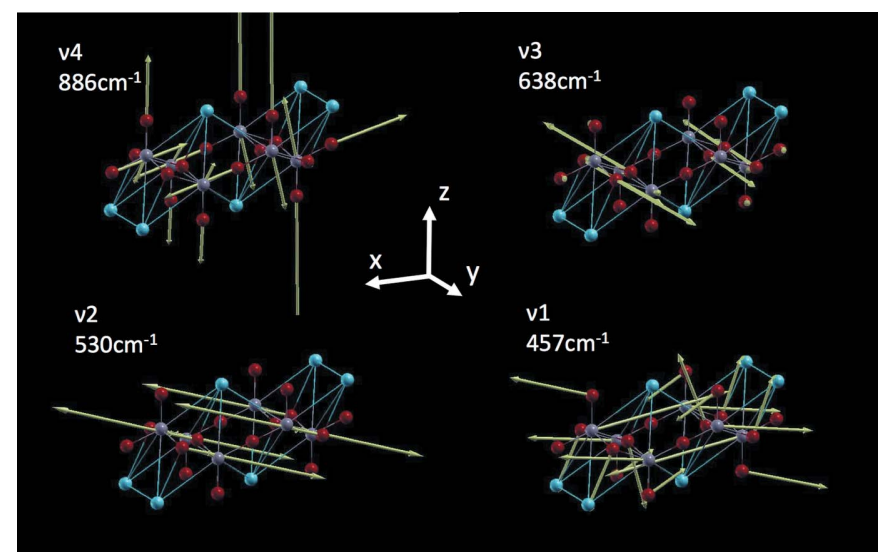

Figure 8

Representations of the vibrational phonon modes $\nu 1, \nu 2, \nu 3$ and $\nu 4$ calculated with density functional theory for $\mathrm{Rb}_{2} \mathrm{Ti}_{2} \mathrm{O}_{5}$, using the generalized gradient approximation (GGA). The other phonon modes are shown in the supporting information.

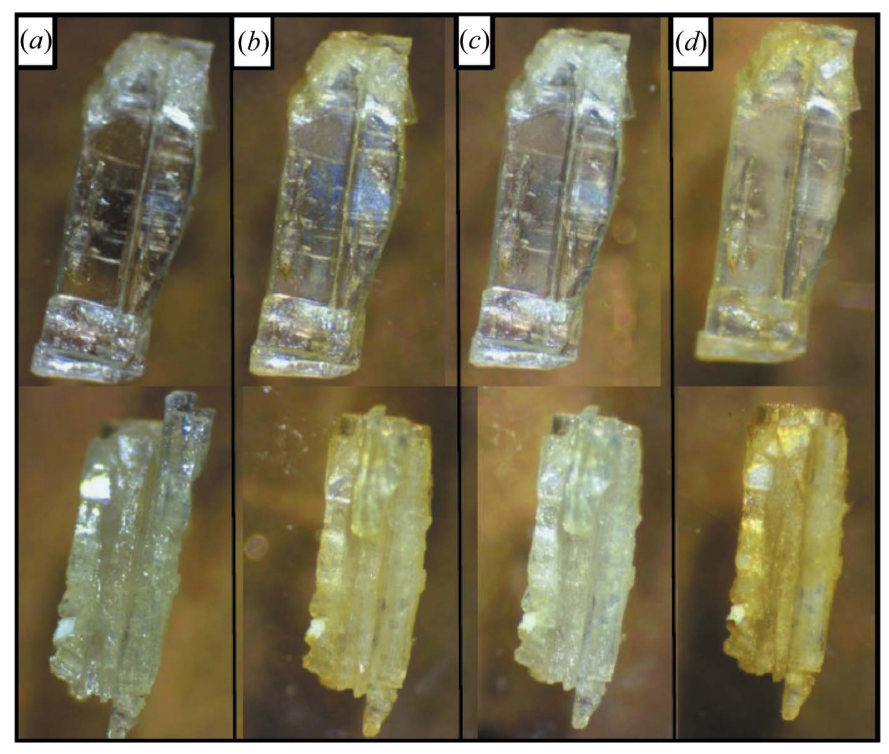

Figure 9

Change of color observed for two different crystals (top and bottom) annealed alternatively under vacuum and oxygen at $400 \mathrm{~K}$ : $(a)$ as-grown, (b) after vacuum annealing for two hours, $(c)$ after oxygen annealing for two hours and $(d)$ after vacuum annealing for two hours. A yellow color is observed after vacuum annealing which indicates the presence of oxygen vacancies. These vacancies seem to be destroyed by oxygen annealing, since a white color is recovered. samples annealed under vacuum at $400 \mathrm{~K}$ for a couple of hours. As a matter of fact, these electrical properties can also be 'deactivated' when the material is annealed under 1 bar oxygen atmosphere at $400 \mathrm{~K}$. This observation tends to indicate that the existence of these electrical properties is correlated with the presence of oxygen vacancies in the material. In addition, Fig. 9 shows that a reversible change of color operates when the material is alternatively annealed under vacuum and oxygen at $400 \mathrm{~K}$. This is a strong indication in favor of the creation of oxygen vacancies since the material turns from white to yellow and yellow to white, similarly to what is observed in $\mathrm{TiO}_{2}$ when oxygen vacancies are created (Sekiya et al., 2000).

A subsequent XRD study has been conducted on RTO powder that was annealed in situ in the diffraction chamber under $\mathrm{N}_{2}$ atmosphere. The corresponding diffractograms are displayed in Fig. 10 and do not show any change as function of the temperature excepted for the appearance of a diffraction peak around $24^{\circ}$ below $250 \mathrm{~K}$. This peak is attributed to a small contamination by water ice. Powder diffraction at room temperature was also performed on samples annealed for $24 \mathrm{~h}$ at $400 \mathrm{~K}$ under vacuum and again, the diffractogram was not modified.

In addition, Raman studies were conducted at $300 \mathrm{~K}$ on the same sample before and after activation. The results are presented in Fig. 11 for the part of the spectrum where four well-identified phonon modes are present. The green spectrum corresponds to the non-activated sample, the blue spectrum to the activated sample. The activation process clearly has the effect of shifting three of the phonon modes

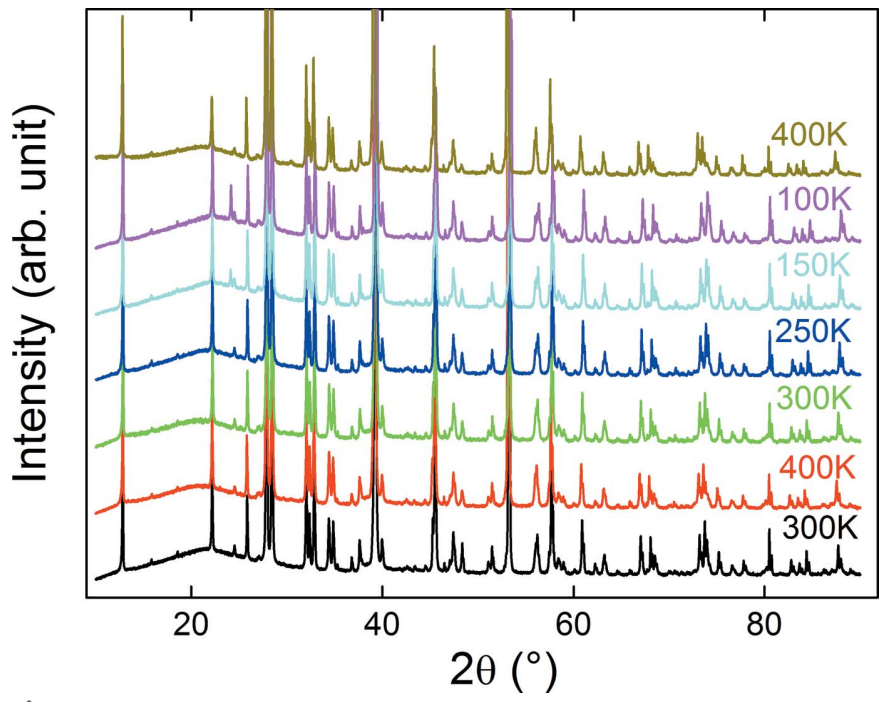

Figure 10

Powder X-ray diffractograms for $\mathrm{Rb}_{2} \mathrm{Ti}_{2} \mathrm{O}_{5}$ taken at different temperatures and displaced for clarity. From bottom to top: as-grown sample measured at $300 \mathrm{~K}$, vacuum-annealed sample measured at $400 \mathrm{~K}$, then at $300 \mathrm{~K}, 250 \mathrm{~K}, 150 \mathrm{~K}, 100 \mathrm{~K}, \mathrm{~N}_{2}$-annealed sample measured at $400 \mathrm{~K}$. All annealing procedures were performed in situ and at $400 \mathrm{~K}$. The annealing durations were $8 \mathrm{~h}$. The peak at $24.17^{\circ}(3.68 \AA)$ comes from the formation of a very thin layer of $\mathrm{H}_{2} \mathrm{O}$ ice on the surface of the sample. Heating the sample from $150 \mathrm{~K}$ up to 250 under vacuum allowed us to reversibly remove the ice and suppress the peak. This indicates that the sample structure is not altered by the presence of residual solid $\mathrm{H}_{2} \mathrm{O}$. 


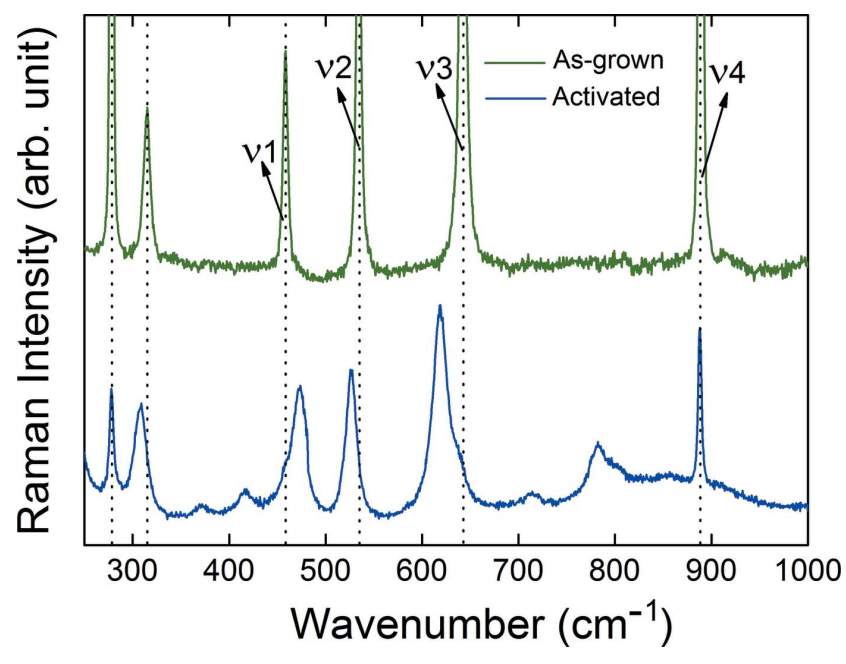

Figure 11

Raman spectra taken at $300 \mathrm{~K}$ for an as-grown sample (green line) and for an activated sample (blue line). (The curves are displaced for clarity.) Note the energy shift produced by the activation process for the $v 1, \nu 2, v 3$ phonon modes whereas $v 4$ remains unaffected. Additional peaks are also produced by the activation.

( $v 1, v 2, v 3$ as represented in Fig. 8), whereas one (v4) remains unaffected. It also produces small additional peaks. This represents an indirect way of probing the microscopic effect of activation. (In particular oxygen vacancies should produce additional peaks as inversion symmetry should be lost.) This, therefore, deserves further investigation, more systematic measurement and DFT simulations taking into account the presence of oxygen vacancies or other defects.

Finally, it is known that X-ray diffraction does not easily discern the presence of oxygen vacancies in a crystal structure with heavy elements. However analysis of some diffuse scattering or of possible satellite reflections (that were not observed with our technique) obtained for instance by synchrotron radiation $\mu \mathrm{XRD}$ as function of temperature might allow detection of a potential order/disorder transition of the oxygen vacancies or interstitial oxygens (Fratini et al., 2010). This will be considered in future experiments. Neutron diffraction will be considered as well in order to study the presence of oxygen vacancies induced by the annealing processes.

\section{Conclusion}

The crystal structure of RTO has been characterized as a function of temperature using X-ray diffraction, electron microscopy and Raman spectroscopy. All methods are consistent with a $C 2 / m$ space group in the whole range of investigated temperature i.e. between $90 \mathrm{~K}$ and $450 \mathrm{~K}$. The outcomes of the X-ray diffraction and Raman spectroscopy are compared to theoretical calculations performed with density functional theory and are in excellent agreement. In particular the computed phonon modes match very well the ones measured experimentally. The structure is also confirmed by transmission electron microscopy.
The special case of the activated material is also inspected by comparing XRD diffractograms of as-grown and annealed samples. While the centrosymmetric $C 2 / m$ structure is found to remain unchanged, further work would be necessary to investigate the possibility of variation of the diffuse scattering with temperature. The comparison between Raman spectra for as-grown and activated materials demonstrates an energy shift of some selective phonon modes that deserves more systematic investigation.

In any case, this work shows that the $C 2 / \mathrm{m}$ structure of the material remains unchanged between $90 \mathrm{~K}$ and $450 \mathrm{~K}$, thus excluding the existence of a structural transition between $200 \mathrm{~K}$ and $330 \mathrm{~K}$. These findings rule out a conventional ferroelectric transition associated to a displacive structural transition as a mechanism responsible for the reported colossal polarizability of RTO. The role of the activation process is still to be investigated.

\section{Acknowledgements}

We thank L. M. Chamoreau for her help during the X-ray diffraction measurements performed on single crystals at the IPCM (Pierre and Marie Curie University, Paris).

\section{Funding information}

This work was supported by French state funds managed by the ANR within the 'Investissements d'Avenir' program under reference ANR-11-IDEX-0004-02, and more specifically within the framework of the Cluster of Excellence MATISSE led by Sorbonne Universités. The project was partly supported by the Region Ile-de-France through a SESAME grant. The authors also acknowledge financial support from the CNRSCEA METSA French network (FR CNRS 3507) on the CLYM platform.

\section{References}

Andersson, S. \& Wadsley, A. D. (1960). Nature, 4736, 499-500.

Andersson, S. \& Wadsley, A. D. (1961). Acta Chem. Scand, 3, 663669.

Baroni, S., de Gironcoli, S., Dal Corso, A. \& Giannozzi, P. (2001). Rev. Mod. Phys. 73, 515-562.

Dolomanov, O. V., Bourhis, L. J., Gildea, R. J., Howard, J. A. K. \& Puschmann, H. (2009). J. Appl. Cryst. 42, 339-341.

Farges F. (1997). Am. Mineral. 82, 44.

Federicci, R., Hole, S., Popa, A. F., Brohan, L., Baptiste, B., Mercone, S. \& Leridon, B. (2017). Phys. Rev. Mater. 1, 21.

Finger, L. W., Cox, D. E. \& Jephcoat, A. P. (1994). J. Appl. Cryst. 27, 892-900.

Foster, M. C., Arbogast, D. J., Nielson, R. M., Photinos, P. \& Abrahams, S. C. (1999). J. Appl. Phys. 85, 2299-2303.

Fratini, M., Poccia, N., Ricci, A., Campi, G., Burghammer, M., Aeppli, G. \& Bianconi, A. (2010). Nature, 466, 841-844.

Giannozzi, P. et al. (2009). J. Phys. Condens. Matter, 21, 395502.

Hohenberg, P. \& Kohn, W. (1964). Phys. Rev. 136, B864-B871.

Kohn, W. \& Sham, L. J. (1965). Phys. Rev. 140, A1133-A1138.

Lii, K.-H. (1995). J. Chem. Soc. Dalton Trans. pp. 927-930.

Momma, K. \& Izumi, F. (2011). J. Appl. Cryst. 44, 1272-1276.

Murnaghan, F. D. (1944). PNAS, 30, 244-247.

Perdew, J. P., Burke, K. \& Ernzerhof, M. (1996). Phys. Rev. Lett. 77, 3865-3868. 
Rabe, K. M. \& Ghosez, P. (2007). First-Principles Studies of Ferroelectric Oxides, pp. 117-174. Berlin, Heidelberg: Springer.

Rietveld, H. M. (1969). J. Appl. Cryst. 2, 65-71.

Rigaku Oxford Diffraction (2015). CrysAlis PRO. Rigaku Oxford Diffraction, Yarnton, England.

Roberts, M. A., Sankar, G., Thomas, J. M. \& Jones, R. H. (1996). Nature, 381, 401-404.

Rodriguez-Carvajal, J. (1993). Phys. B: Condens. Matter, 192, 55-69.

Schmitz-DuMont, O. \& Reckhard, H. (1959). Monatsh. Chem. 90, 134-142.

Schmitz-Dumont, O. \& Schulz, A. H. (1952). Monatsh. Chem. 83, 638-649.

Sekiya, T., Ichimura, K., Igarashi, M. \& Kurita, S. (2000). J. Phys. Chem. Solids, 61, 1237-1242.
Setyawan, W. \& Curtarolo, S. (2010). Comput. Mater. Sci. 49, 299312.

Sheldrick, G. M. (2015). Acta Cryst. A71, 3-8.

Sheldrick, G. M. (2015). Acta Cryst. C71, 3-8.

Shripal, Badhwar, S., Maurya, D. \& Kumar, J. (2005). J. Mater. Sci. Mater. Electron. 16, 495-500.

Tournoux, M., Marchand, R. \& Brohan, L. (1986). Prog. Solid State Chem. 17, 33-52.

Vikram, S. V., Phase, D. M. \& Chandel, V. S. (2010). J. Mater. Sci. Mater. Electron. 21, 902-905.

Wang, Q., Guo, Z. \& Chung, J. S. (2009). Chem. Commun. 35, 52845286.

Wang, Q., Chung, J. S. \& Guo, Z. (2011). Ind. Eng. Chem. Res. 50, 8384-8388. 